\title{
Obstruction à la transversalité de lagrangiens
}

\author{
Max Karoubi ${ }^{\mathrm{a}}$, Maria Luiza Lapa de Souza ${ }^{\mathrm{b}}$ \\ a Département de mathématiques, UMR 7586 du CNRS, case 7012, université Paris 7, 2, place Jussieu, 75251 Paris cedex 05, France \\ ${ }^{\mathrm{b}}$ Instituto de Matemática, Universidade Federal da Bahia, Campus de Ondina, 40170.100, Ondina, SSA/Bahia, Brasil
}

Reçu le 8 octobre 2005 ; accepté après révision le $1^{\mathrm{er}}$ mars 2006

Disponible sur Internet le 18 avril 2006

Présenté par Étienne Ghys

\section{Résumé}

Dans cette Note, nous donnons une condition nécessaire et suffisante pour que plusieurs lagrangiens dans un fibré vectoriel symplectique puissent être déformés stablement en lagrangiens transversaux. Dans le cas de trois lagrangiens, nous montrons que le groupe de Grothendieck associé à ce problème s'identifie à un groupe de K-théorie hermitienne. Pour citer cet article:M. Karoubi, M.L. Lapa de Souza, C. R. Acad. Sci. Paris, Ser. I 342 (2006).

@ 2006 Académie des sciences. Publié par Elsevier SAS. Tous droits réservés.

\section{Abstract}

Obstruction to Lagrangian transversality. In this Note, we give a necessary and sufficient condition for Lagrangians in a symplectic vector bundle to be deformed stably into transversal Lagrangians. In the case of three Lagrangians, we show that the associated Grothendieck group can be identified with a Hermitian K-theory group. To cite this article: M. Karoubi, M.L. Lapa de Souza, C. R. Acad. Sci. Paris, Ser. I 342 (2006).

() 2006 Académie des sciences. Publié par Elsevier SAS. Tous droits réservés.

1. Soit $E$ un fibré vectoriel réel de base $X$ muni d'une forme symplectique non dégénérée (dite aussi (-1)-hermitienne). Un sous-fibré $L$ est dit lagrangien si son orthogonal est égal à L. Deux lagrangiens $L_{1}$ et $L_{2}$ sont transversaux si leur intersection $L_{1} \cap L_{2}$ est réduite à 0 . Ils sont homotopiquement transversaux s'il existe deux familles continues de lagrangiens $L_{1}(\mathrm{t})$ et $L_{2}(\mathrm{t})$ telles que $L_{1}(0)=L_{1}, L_{2}(0)=L_{2}$, les lagrangiens $L_{1}(1)$ et $L_{2}(1)$ étant transversaux. Enfin, $L_{1}$ et $L_{2}$ sont dits stablement et homotopiquement transversaux si $L_{1} \oplus L$ et $L_{2} \oplus L$ sont homotopiquement transversaux dans le fibré $E \oplus H(L)$, où $H(L)=L \oplus L^{*}$ désigne le fibré hyperbolique associé à $L$.

2. Considérons le monoï de (pour la somme directe) des triplets $\left(E, L_{1}, L_{2}\right)$, où $E$ est un fibré symplectique avec deux lagrangiens $L_{1}$ et $L_{2}$. Un tel triplet est élémentaire si $L_{1}=L_{2}$. Deux triplets $\sigma$ et $\sigma^{\prime}$ sont équivalents s'il existe $\tau$ et $\tau^{\prime}$ élémentaires tels que $\sigma+\tau$ soit isomorphe à $\sigma^{\prime}+\tau^{\prime}$ en un sens évident. L'ensemble des classes d'équivalence forme un groupe $U(X)$ étudié dans [1] et [2] dans un contexte plus général. On note $d\left(E, L_{1}, L_{2}\right)$ la classe du triplet

Adresses e-mail : karoubi@math.jussieu.fr (M. Karoubi), lapa@noos.fr (M.L. Lapa de Souza). 
$\left(E, L_{1}, L_{2}\right)$ dans $U(X)$. Ce groupe $U(X)$ s'insère dans une suite exacte entre des groupes de K-théorie réelle et complexe, où la dernière flèche est induite par la complexification des fibrés

$$
K_{R}^{-1}(X) \rightarrow K_{C}^{-1}(X) \rightarrow U(X) \rightarrow K_{R}(X) \rightarrow K_{C}(X) .
$$

En fait, on démontre dans [1] que $U(X)$ s'identifie à un certain groupe $V^{1}(X)$ obtenu à partir de triplets $\left(F, g_{1}, g_{2}\right)$, où $F$ est un fibré réel (sur une 7-ième-suspension de $X^{+}$) et où $g_{1}$ et $g_{2}$ sont deux formes quadratiques non dégénérées sur $F$. Plus précisément, $U(X) \cong V^{1}(X)$ est aussi isomorphe à $K_{R}^{1}(X)$ qui est la K-théorie associée à la catégorie des fibrés vectoriels réels sur la 7-ième suspension topologique de $X^{+}$(ceci est une version réelle de la périodicité de Bott).

Dans sa thèse [5], le deuxième auteur a calculé l'obstruction à la transversalité homotopique stable des lagrangiens $L_{1}$ et $L_{2}$, problème initialement posé au début. Elle se situe dans le conoyau de la flèche $K_{R}(X) \rightarrow U(X)$ qui associe à un fibré $L$ la classe du triplet $\left(H(L), L, L^{*}\right)$. En raison de la périodicité de Bott de nouveau, on montre que cette flèche est nulle. L'obstruction cherchée appartient donc simplement au groupe $U(X) \cong K_{R}^{1}(X)$. Comme il est montré dans [5], l'invariant de Maslov classique associé à deux lagrangiens se déduit de cet invariant.

\section{3.}

Remarque. Si $Q$ est le corps des nombres rationnels, le groupe $K_{R}^{1}(X) \otimes Q$ s'identifie à la somme des groupes de cohomologie de Cech $H^{4 n+1}(X ; Q)$.

4. Examinons maintenant la situation de trois lagrangiens $L_{1}, L_{2}$ et $L_{3}$ avec une extension évidente des définitions de transversalité.

Théorème 1. Pour que trois lagrangiens $L_{1}, L_{2}$ et $L_{3}$ soient homotopiquement et stablement transversaux, il faut et il suffit que $d\left(E, L_{1}, L_{2}\right)=d\left(E, L_{1}, L_{3}\right)=0$ dans le groupe $U(X)$ (ce qui implique aussi $\left.d\left(E, L_{2}, L_{3}\right)=0\right)$.

Démonstration. La condition étant évidemment nécessaire, démontrons qu'elle est suffisante. Puisque $d\left(E, L_{1}, L_{2}\right)$ $=0$, on peut supposer (stablement) que $E=H(L)$ avec $L_{2}=L$ et $L_{1}=L^{*}$ qui est isomorphe à $L$ en tant que fibré réel par le choix d'une métrique sur $L$. Par ailleurs, puisque $d\left(E, L_{2}, L_{3}\right)=0$, on peut aussi supposer que $L_{3}$ est transverse à $L_{2}$ et qu'il est défini par le graphe d'un morphisme $g: L=L_{1} \rightarrow L^{*}=L_{2}$. Puisque $L_{3}$ est un lagrangien dans un fibré symplectique, on a nécessairement $g^{*}=g$. Soit maintenant $h$ une métrique sur $L$ définissant donc un isomorphisme auto-adjoint entre $L$ et $L^{*}$. Alors le graphe de l'endomorphisme auto-adjoint $g(t)=(1-t) g+t h$ définit une homotopie entre $L_{3}$ et un lagrangien transversal à la fois à $L_{1}$ et $L_{2}$ (pour $t=1$ ).

5. La même méthode permet de démontrer le théorème plus général suivant :

Théorème 2. Soit $E$ un fibré symplectique et soient $L_{1}, \ldots, L_{m}$ des lagrangiens avec $m \geqslant 2$. Pour que ceux-ci soient homotopiquement et stablement transversaux, il faut et il suffit que dans le groupe $U(X)$ on ait $d\left(E, L_{1}, L_{n}\right)=0$ pour tout $n$ (ce qui implique $d\left(E, L_{i}, L_{j}\right)=0$ pour tout couple $(i, j)$ ).

Démonstration. Le raisonnement précédent montre que les $L_{n}, n \geqslant 3$, peuvent être associés (à homotopie près) à des métriques $h_{n}$ sur $L=L_{1}$. Par homothétie, on peut même supposer que $h_{n}=n h$, où $h$ est une métrique fixe sur $L$. On en déduit que $h_{n}-h_{p}=(n-p) h$ est inversible pour $n \neq p$ et et que donc $L_{n}$ est transversal à $L_{p}$.

6. Nous allons voir que la situation est légèrement différente pour des fibrés $E$ munis d'une forme bilinéaire symétrique non dégénérée (ou encore $(+1)$-hermitienne). Dans ce cas, le groupe $U(X)$ construit précédemment avec des couples de lagrangiens est isomorphe à $K_{R}^{-1}(X)$ d'après [1]. L'obstruction pour la transversalité homotopique stable de deux lagrangiens appartient alors au conoyau de la flèche $K_{R}(X) \rightarrow K_{R}^{-1}(X)$ qui s'insère dans la suite exacte de Bott (où la dernière flèche est induite par la réalification des fibrés) :

$$
K_{R}(X) \rightarrow K_{R}^{-1}(X) \rightarrow K_{C}^{-1}(X) \rightarrow K_{R}^{1}(X) .
$$

L'obstruction à la transversalité de $L_{1}$ et $L_{2}$ peut donc être considérée comme un élément du groupe $N=$ $\operatorname{Ker}\left[K_{C}^{-1}(X) \rightarrow K_{R}^{1}(X)\right]$, élément qu'on notera $\gamma\left(E, L_{1}, L_{2}\right)$. 
7.

Remarque. Comme dans le paragraphe 3, on peut montrer que le groupe $N \otimes Q$ s'identifie à la somme des groupes de cohomologie de Cech $H^{4 n-1}(X ; Q)$.

8. Soient $L_{1}, L_{2}$ et $L_{3}$ trois lagrangiens tels que $L_{1}$ et $L_{2}$ d'une part, $L_{2}$ et $L_{3}$ d'autre part, soient homotopiquement et stablement transversaux. Donc $L_{3}$ est défini par un morphisme de fibrés $h: L=L_{1} \rightarrow L^{*}=L_{2}$ tel que $h^{*}=-h$. Pour que $L_{3}$ soit homotopiquement transversal à $L_{2}$, il faut et il suffit donc que $L$ puisse être muni d'une structure symplectique non dégénérée (ce qui est équivalent à une structure complexe). Puisqu'on raisonne stablement, on peut remplacer $E$ par $E \oplus H(L)$, et donc $L$ par $L \oplus L$. Sans restreindre la généralité, on peut ainsi supposer que $L$ est bien muni d'une structure symplectique non dégénérée. On en déduit le théorème suivant, dont la démonstration est calquée sur celle du théorème précédent (en remplaçant les métriques par des formes symplectiques non dégénérées).

Théorème 3. Soit $E$ un fibré vectoriel muni d'une forme bilinéaire symétrique non dégénérée et soient $L_{1}, \ldots, L_{n}$ des lagrangiens dans E. Pour que ces lagrangiens soient homotopiquement et stablement transversaux, il faut et il suffit que $\gamma\left(E, L_{1}, L_{n}\right)=0$ pour tout $n$ (ce qui implique $\gamma\left(L_{1}, L_{i}, L_{j}\right)=0$ pour tout couple $(i, j)$ ).

9.

Remarque importante. Il est facile de voir que la théorie $U(X)$ est un foncteur semi-exact dont l'espace classifiant est le groupe orthogonal infini $O=\operatorname{colim} O(n)$ dans le cas $(+1)$-hermitien ou l'espace homogène infini $U / O=\operatorname{colim} U(n) / O(n)$ dans le cas $(-1)$-hermitien. Si $X$ est un CW-complexe de dimension $N$, la transversalité homotopique stable est donc équivalente à la transversalité homotopique si $n>N+1$ dans le premier cas et $n>N+2$ dans le second ( $n$ étant le rang du fibré $E$ ).

10. Revenons au cas d'un fibré symplectique $E$ muni de trois lagrangiens $\left(L_{1}, L_{2}, L_{3}\right)$. Sur le fibré $L_{1} \oplus L_{2} \oplus L_{3}$ on peut définir la forme quadratique suivante

$$
q\left(x_{1}, x_{2}, x_{3}\right)=\phi\left(x_{1}, x_{2}\right)+\phi\left(x_{2}, x_{3}\right)+\phi\left(x_{3}, x_{1}\right)
$$

utilisée notamment par Leray [7] et Kashiwara [4].

Théorème 4. Les trois lagrangiens $L_{1}, L_{2}$ et $L_{3}$ sont transversaux si et seulement si la forme quadratique q est non dégénérée.

Démonstration. Supposons d'abord que les trois lagrangiens ne soient pas transversaux, par exemple que $L_{1} \cap L_{2} \neq$ 0 . Choisissons un élément non nul $y_{1}=y_{2} \in L_{1} \cap L_{2}$. Posons aussi $y_{3}=0$. Pour tout triplet $\left(x_{1}, x_{2}, x_{3}\right)$, calculons alors la forme bilinéaire symétrique associée à $q$, soit

$$
\Psi(x, y)=\phi\left(x_{1}, y_{2}\right)-\phi\left(y_{1}, x_{2}\right)+\phi\left(x_{2}, y_{3}\right)-\phi\left(y_{2}, x_{3}\right)+\phi\left(x_{3}, y_{1}\right)-\phi\left(y_{3}, x_{1}\right) .
$$

Elle se réduit à $\phi\left(x_{3}, y_{1}\right)-\phi\left(x_{3}, y_{2}\right)+\phi\left(y_{3}, x_{1}\right)=\phi\left(x_{3}, y_{1}-y_{2}\right)+\phi\left(y_{3}, x_{1}\right)=0$. Ceci montre que l'orthogonal de $F=L_{1} \oplus L_{2} \oplus L_{3}$ pour la forme de Leray-Kashiwara n'est pas nul et que la forme quadratique $q$ est dégénérée.

Réciproquement, supposons que les lagrangiens soient transversaux. On peut alors poser $L_{1}=L, L_{2}=L^{*}, L_{3}$ étant défini comme le graphe d'un morphisme $g: L \rightarrow L^{*}$ tel que $g^{*}=g$. Considérons le sous-espace $E_{3}$ de $\mathrm{F}$ formé des triplets $\left(x_{1}, x_{2}, x_{3}\right)$ tels que $x_{1}+x_{2}-x_{3}=0$. Si on pose $x_{3}=(u, g u)$ dans l'expression de $L_{3}$ comme sous-espace de $L_{1} \oplus L_{2}$, on voit que $E_{3}$ est isomorphe à $L_{1}$. Pour calculer la forme $\Psi$ restreinte à $E_{3}$, on pose alors $x_{1}=x_{1}^{\prime}=u$, $x_{2}=x_{2}^{\prime}=g u, y_{1}=y_{1}^{\prime}=v, y_{2}=y_{2}^{\prime}=g v$.

En appliquant la formule $(F)$ ci-dessus, on voit que la forme $\Psi$ restreinte à $E_{3}$ s'écrit de la manière suivante en fonction de $u$ et $v \in L_{1} \cong E_{3}$, le symbole $\langle$,$\rangle désignant l'accouplement entre un espace et son dual :$

$$
\begin{aligned}
\Psi(u, v) & =\langle u, g v\rangle-\langle v, g u\rangle+\langle v, g u\rangle-\langle u, g v\rangle+\langle v, g u\rangle-\langle u, g v\rangle \\
& =-2\langle u, g v\rangle .
\end{aligned}
$$


Ainsi, au facteur -2 près, la forme bilinéaire symétrique sur $E_{3}$ s'identifie à la forme bilinéaire symétrique associée à $g$ (modulo l'identification entre $E_{3}$ et $L_{1}$ ). Par ailleurs, si on considère de nouveau la formule $(F)$ ci-dessus, on voit que l'orthogonal de $H\left(L_{1}\right)=L_{1} \oplus L_{2}$ dans $F=L_{1} \oplus L_{2} \oplus L_{3}$ est formé des triplets $\left(y_{1}, y_{2}, y_{3}\right)$, avec $y_{3}=\left(y_{1}^{\prime}, y_{2}^{\prime}\right)$ tels que pour tout couple $\left(x_{1}, x_{2}\right)$, on ait $\left\langle x_{1}, y_{2}-y_{2}^{\prime}\right)+\left\langle y_{1}-y_{1}^{\prime}, x_{2}\right\rangle=0$. Donc $y_{1}^{\prime}=y_{1}$ et $y_{2}^{\prime}=y_{2}$, ce qui détermine bien le sous-module $E_{3}$. Par conséquent, $F$ s'identifie à la somme orthogonale de $E_{3}$ et de $H\left(L_{1}\right)$ : ceci démontre que la forme quadratique q est non dégénérée.

11. Considérons maintenant le monoïde des classes d'isomorphie des quadruplets précédents $\left(E, L_{1}, L_{2}, L_{3}\right)$ où $L_{1}, L_{2}$ et $L_{3}$ sont des lagrangiens transversaux. Notons $L K(X)$ le groupe de Grothendieck associé à ce monoïde (en référence au travail de Leray et Kashiwara).

Théorème 5. Le groupe $L K(X)$ s'identifie au groupe de $K$-théorie hermitienne de l'espace $X$, c'est-à-dire à la somme directe de deux copies de la $K$-théorie réelle [3]. De manière précise, l'isomorphisme

$$
L K(X) \rightarrow K_{R}(X) \oplus K_{R}(X)
$$

est induit par la correspondance

$$
\left(E, L_{1}, L_{2}, L_{3}\right) \mapsto[\Psi]-H\left(L_{1}\right)
$$

où $\Psi$ est la forme quadratique de Leray-Kashiwara.

Démonstration. D'après ce qui précède, les classes d'isomorphie des quadruplets précédents sont en correspondance bijective avec les formes bilinéaires symétriques non dégénérées $g: L_{1} \rightarrow L_{1}^{*}$. Le théorème résulte alors des considérations du paragraphe 10 .

12. Généralisations diverses. Pour simplifier, nous nous sommes placés dans le cadre géométrique des fibrés vectoriels. Les considérations précédentes se généralisent sans peine au cadre des modules projectifs de type fini sur une $C^{*}$-algèbre quelconque. Une autre généralisation est de considérer des fibrés équivariants [6]. Les résultats démontrés dans cette Note s'étendent aussi sans problème à ce cadre.

\section{Références}

[1] M. Karoubi, Périodicité de la K-théorie hermitienne, in : Lecture Notes in Math., vol. 343, Springer, 1973, pp. $301-411$.

[2] M. Karoubi, Le théorème fondamental de la K-théorie hermitienne, Ann. Math. 112 (1980) 559-582.

[3] M. Karoubi, K-Theory. An Introduction, Springer-Verlag, 1978, (p. 47, exercice 9.22).

[4] M. Kashiwara, P. Schapira, Sheaves on Manifolds, in: Grundlehren Math. Wiss., vol. 292, Springer-Verlag, 1994 (with a chapter in French by Christian Houzel).

[5] M.L. Lapa de Souza, Interprétation de la classe de Maslov dans la K-théorie hermitienne et dans la théorie relative de Chern-Weil, K-Theory 13 (1998) 347-361.

[6] M.L. Lapa de Souza, Les invariants de Maslov équivariants, K-Theory 25 (2002) 233-259.

[7] J. Leray, Analyse lagrangienne et mécanique quantique, in: Séminaire sur les Équations aux Dérivées Partielles (1976-1977), I, Exp. No. 1, Collège de France, Paris, 1977. 